\title{
Small-Animal PET Imaging of Isolated Perfused Rat Heart
}

\author{
Tomohiko Yamane ${ }^{1}$, Min-Jae Park ${ }^{2}$, Dominik Richter ${ }^{1}$, Stephan G. Nekolla ${ }^{3}$, Mehrbod S. Javadi ${ }^{4}$, Constantin Lapa ${ }^{1}$, \\ Samuel Samnick ${ }^{1}$, Andreas K. Buck ${ }^{1}$, Ken Herrmann ${ }^{1}$, and Takahiro Higuchi ${ }^{1,2}$ \\ ${ }^{1}$ Department of Nuclear Medicine, University of Würzburg, Würzburg, Germany; ${ }^{2}$ Comprehensive Heart Failure Center, University of \\ Würzburg, Würzburg, Germany; ${ }^{3}$ Nuklearmedizinische Klinik und Poliklinik, Klinikum rechts der Isar, Technische Universität \\ München, Munich, Germany; and ${ }^{4}$ Division of Nuclear Medicine, Russell H. Morgan Department of Radiology, Johns Hopkins \\ University, Baltimore, Maryland
}

The assessment of myocardial radiotracer kinetics, including cardiac extraction fraction and washout, requires the study of isolated perfused hearts to avoid analytic error due to tracer recirculation and systemic metabolites. Analysis of the isolated perfused rat heart by a high-resolution small-animal PET system may offer both reliable evaluation of cardiac tracer kinetics and tomographic images. Methods: An isolated perfused heart system was modified to accommodate the small PET gantry bore size. Isolated rat hearts were perfused via the Langendorff method under a constant flow of Krebs-Henseleit buffer containing ${ }^{18} \mathrm{~F}-\mathrm{FDG}$ with a rate of $5 \mathrm{~mL} / \mathrm{min}$ and placed in the field of view of the commercially available small-animal PET system. Dynamic PET imaging was then performed, with ${ }^{18} \mathrm{~F}-\mathrm{FDG}$ uptake in the isolated perfused heart verified by $\gamma$ counter measurements. Additionally, a rat heart of myocardial infarction was also studied in this system with static PET imaging. Results: Dynamic PET acquisition of the isolated heart under constant ${ }^{18} \mathrm{~F}-\mathrm{FDG}$ infusion demonstrated continuous increase of activity in the heart. Correlation between cardiac activity $(\mathrm{MBq})$ measured with the PET system and measurements made with the $\gamma$ counter were excellent $\left(R^{2}=\right.$ $0.98, P<0.001, n=10)$. Tracer input rate $(\mathrm{MBq} / \mathrm{min})$ was also well correlated with cardiac tracer uptake rate $(\mathrm{MBq} / \mathrm{min})\left(R^{2}=0.87\right.$, $P<0.001, n=12$ ). PET imaging of the heart with myocardial infarction showed a clear tracer uptake defect corresponding to the location of scar tissue identified by autoradiography and histology. Conclusion: Combining the Langendorff method of isolated rat heart perfusion with high-resolution small-animal PET allows for the reliable quantification of myocardial tracer kinetics. This novel assay is readily adapted to available small-animal PET systems and may be useful for understanding myocardial PET tracer kinetics.

Key Words: isolated perfused heart; PET; rat heart; myocardial infarction

J Nucl Med 2014; 55:495-499

DOI: 10.2967/jnumed.113.129429

$\mathbf{N}$ oninvasive high-resolution cardiac PET is a promising imaging technique for advancing our understanding of heart disease (1). Absolute quantification of biologic parameters has been proposed with in vivo PET imaging using dynamic acquisition and

\footnotetext{
Received Jul. 16, 2013; revision accepted Oct. 17, 2013.

For correspondence contact: Takahiro Higuchi, Comprehensive Heart Failure Center/Department of Nuclear Medicine, University of Würzburg, Oberduerrbacher Strasse 6, 97080 Würzburg, Germany.

E-mail: higuchi_t@ukw.de

Published online Jan. 30, 2014.

COPYRIGHT (C 2014 by the Society of Nuclear Medicine and Molecular Imaging, Inc.
}

compartment model kinetic analysis. An assay for quantifying the kinetic properties of PET tracers ex vivo is an important step to a refined understanding of the in vivo molecular and cellular events involved in the genesis and progression of heart disease (2). As such, to further understand the mechanisms and pathways of PET tracers in the heart, kinetic parameters including initial tracer extraction (first-pass extraction) and tissue retention must first be determined with and without target blocking experiments. An isolated perfused heart system (e.g., the Langendorff heart system, which originated in 1895 (3)) is the standard approach that allows the determination of these kinetic parameters without systemic interference such as tracer recirculation and interference by systemic metabolites (4-10).

In-house custom-made dedicated coincidence $\gamma$ detectors are the most commonly used counting systems for measuring PET tracer activity in isolated perfused heart studies (6-9). The major disadvantages of such systems are the time and effort requirements and the inability to provide regional kinetic analysis. These systems can be used only for isolated heart studies and measurement of total heart counts; no tomographic or regional data are acquired.

Recently, several small-animal PET systems have been introduced, allowing for high-resolution preclinical small-rodent PET imaging. These systems have better temporal and spatial resolution than the clinical PET systems adapted for small-animal use (11-13). Because these small-animal PET imaging systems are designed and dedicated for in vivo tomography of smallanimal organs, reliable measurement of tracer activity in rodent hearts are feasible (14). Furthermore, these systems not only allow for global cardiac tracer activity measurement, but also provide tomographic regional data of tracer distribution. To combine the isolated perfused rodent heart study and small-animal PET imaging system, some modifications of the apparatus must be made. Most importantly, the design of the isolated perfused heart module, including the heat water tube, buffer tube, and drip-collecting system, must be modified to accommodate a smaller mounting space. The gantry bore opening of most small-animal PET systems is quite small, measuring typically $9-13 \mathrm{~cm}$ in diameter (11-13).

The purpose of this study was to develop a unified system and demonstrate the feasibility of imaging an isolated perfused rat heart with a high-resolution small-animal PET imaging device.

\section{MATERIALS AND METHODS}

\section{Animals}

The hearts used in the isolated perfused study were harvested from male Wistar rats (200-250 g, Charles River Laboratories International Inc.). Experimental protocols were approved by the regional governmental commission of animal protection (Germany) and are in 
compliance with the Guide for the Care and Use of Laboratory Animals (15).

\section{Isolated Perfused Heart (Constant-Flow Langendorff Method)}

After heparinization of the rats, the hearts were extirpated under deep anesthesia with $2 \%$ isoflurane, quickly cannulated via the aorta, and perfused with standard Krebs-Henseleit $(\mathrm{KH})$ buffer under a constant flow with a rate of $5 \mathrm{~mL} / \mathrm{min}$. The $\mathrm{KH}$ buffer was oxygenated more than $1 \mathrm{~h}$ with carbogen (95\% oxygen and 5\% carbon dioxide) and kept at a temperature of $38.0 \pm 1.0^{\circ} \mathrm{C}$ and $\mathrm{pH}$ of $7.1 \pm 0.1$ at the point where the buffer flows into the heart. Plastic paraffin films (Parafilm; Pechiney Plastic Packaging, Inc.) were covered to avoid drying and fluttering of the hearts.

\section{Small-Animal PET System for Isolated Perfused Heart Study}

Figure 1 shows a photograph and schematic diagram of the combination of isolated perfused rat heart and small-animal PET systems used in the present study. The isolated perfused heart system, including the isolated heart, hot water tube, buffer tube, and drip-collecting system, is designed to fit within the gantry bore opening $(12 \mathrm{~cm})$ of our small-animal PET scanner (Inveon; Siemens Medical Solutions). Further technical specifications are given in the supplemental data (available at http://jnm.snmjournals.org).

\section{PET Image Acquisition, Processing, and Data Analysis}

After confirmation of stable contraction, the isolated hearts were placed within the field of view of the PET system. Then, KH buffer infusion containing radionuclide tracer was initiated, and PET data were acquired in list-mode. The raw data were sorted into $2 \mathrm{~min} / \mathrm{frames}$ and reconstructed by ordered-subset expectation maximization with 16 subsets and 4 iterations. The reconstructed PET images were analyzed using an image-processing application (AMIDE-bin 1.0.2) (16). Regions of interest were manually drawn to cover the entire cardiac activity, and time-activity curves were generated. As a standard reference for quantification of the isolated heart counts, a cylindric small 1.4-mL phantom containing ${ }^{18} \mathrm{~F}$ with known activity was measured by both PET and $\gamma$ well counter.

\section{Validation of Small-Animal PET Measurement of Isolated Heart}

Quantification of Tracer Activity in Isolated Perfused Heart. Isolated hearts $(n=10)$ were perfused with $\mathrm{KH}$ buffer containing variable concentrations of ${ }^{18} \mathrm{~F}-\mathrm{FDG}(0.1-0.4 \mathrm{MBq} / \mathrm{mL})$. Immediately after the PET imaging session, the isolated perfused heart was removed from the Langendorff system for tissue activity measurements using a $\gamma$ well counter (CRC-10R; Capintec, Inc.). These values were used for comparison analysis with those acquired during the PET study (last 2-min frame).

Measurement of Tracer Uptake Rate. Isolated hearts $(n=6)$ were perfused with $\mathrm{KH}$ buffer containing variable concentrations $(\mathrm{MBq} / \mathrm{mL})$ of ${ }^{18} \mathrm{~F}$-FDG. Two different concentrations in each heart (total, 12 different concentrations) were analyzed. The slope of the time-activity curve $(\mathrm{MBq} / \mathrm{min})$ acquired by dynamic PET imaging data was calculated by linear fitting of 5 serial time points ( $2 \mathrm{~min} / \mathrm{frame})$.

Rat Model of Coronary Occlusion. One rat heart with myocardial infarction was studied by our unified isolated perfused heart and smallanimal PET imaging system. Additionally, reference in vivo PET imaging, ex vivo autoradiography, and histologic analysis with hematoxylin and eosin stain were performed. The myocardial infarction was induced by ligating the left coronary artery with a 7-0 polypropylene suture via a left lateral thoracotomy (17). One week after the operation, static 10-min in vivo PET imaging was performed $30 \mathrm{~min}$ after intravenous administration of $37 \mathrm{MBq}$ of ${ }^{18} \mathrm{~F}-\mathrm{FDG}$. After $2 \mathrm{~d}$ of ${ }^{18} \mathrm{~F}$ decay, the same rats were used for isolated perfused heart imaging. ${ }^{18}$ F-FDG PET imaging was performed with a static 20 -min acquisition. Immediately after the scan, the heart was frozen and embedded in Tissue-Tek O.C.T. compound (Sakura Finetek Europe). Horizontal long-axis tissue sections with $20-\mu \mathrm{m}$ thicknesses were obtained using a cryostat for autoradiography and histology. The sections were exposed for $1 \mathrm{~h}$ on a phosphor imaging plate, and digital autoradiographic images were obtained (CR 35 Bio; Raytest). Finally, the sections were stained with hemotoxylin and eosin.

\section{Statistical Analysis}

Data were expressed with mean and SD. Results of PET imaging and $\gamma$ well counter data were evaluated by linear regression analysis, and a $P$ value of less than 0.05 was considered to be statistically significant.

\section{RESULTS}

Combination of Small-Animal PET and Isolated Perfused Heart

We modified an isolated perfused heart apparatus for smallanimal PET imaging. The major components including the buffer reservoir, hot water sink, pump system, and monitoring system were placed on a mobile table alongside the small-animal PET system. Compact components of the isolated heart chamber, including the warm water tubing system, perfusion medium transport tubing, aortic cannula, drip-collecting system, and drainage systems, were placed in the field of view of the PET scanner (Fig. 1). We observed stable beating of the rat hearts during PET acquisition $(190 \pm 14 \mathrm{bpm}$ at the start of scan vs. $182 \pm 16 \mathrm{bpm}$ at the end of scan, not significant).

\section{Activity of Isolated Perfused Heart Measured by Small-Animal PET System}

Under constant ${ }^{18} \mathrm{~F}$-FDG infusion, activPET gantry ity in the isolated heart increased steadily as measured by the small-animal PET system. The tracer activity at the last frame estimated by PET showed excellent correlation with the gold standard measured by the $\gamma$ well counter, indicating the high accuracy of the
FIGURE 1. Photograph of combination of isolated perfused rat heart apparatus and smallanimal PET system (A) and schematic diagram illustrating isolated perfused heart system placed in gantry bore of small-animal PET scanner (B). 


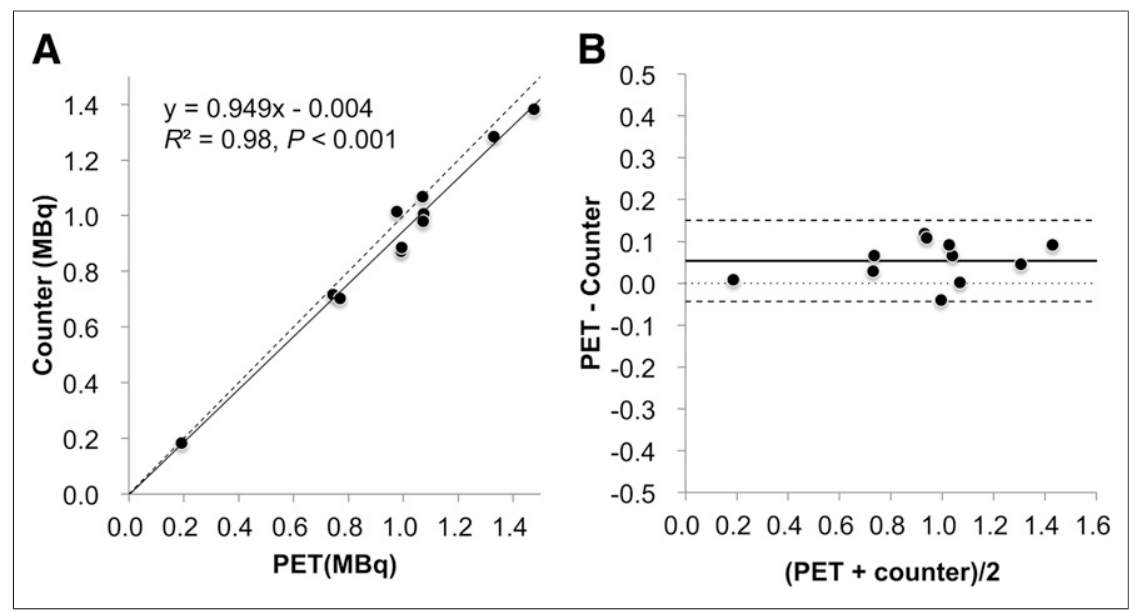

FIGURE 2. Linear regression plot (A) and Bland-Altman plot (B) of correlation analysis between activities of isolated perfused heart measured by PET and $\gamma$ well counter. In linear regression plot, solid line indicates regression, and dashed line indicates where indices of PET and well counter are identical $(y=x)$. In Bland-Altman plot, solid line indicates mean bias, dashed line indicates mean \pm 1.96 SDs, and dotted line indicates line of no bias.

PET approach (Fig. 2). Furthermore, with varying concentrations of ${ }^{18} \mathrm{~F}$-FDG, we again observed excellent correlation between the uptake rate by the PET approach and tracer input rate measured by the $\gamma$ counter (Fig. 3).

\section{Isolated Perfused Heart with Myocardial Infarction}

A heart at $1 \mathrm{wk}$ after left coronary occlusion was studied to confirm the feasibility of tomographic imaging of the isolated perfused heart. The reconstructed images of the heart showed a clear defect of tracer uptake at the apex corresponding with the location of scar tissue identified by in vivo PET imaging, autoradiography, and histology (Fig. 4).

\section{DISCUSSION}

This study proposes a novel application of small-animal PET imaging systems for tracer kinetic analysis in isolated perfused rat heart experiments. Although some modification of the isolated perfused heart apparatus is required to accommodate the small size of the small-animal PET gantry (12-cm bore opening), this approach is readily feasible using commercially available in vivo small-animal PET imaging systems. Because these PET systems provide 3-dimensional volumetric information, this approach improves the standard isolated perfused heart apparatus in which only global tracer kinetics can be measured. Our approach allows for complete tomographic analysis of the isolated perfused rat heart without interference by background contamination. This is critical when analyzing the regional tracer kinetics of damaged myocardium in rat myocardial infarction models. Tracer heterogeneity can be clearly defined ex vivo with this system, allowing for segmental kinetic quantification.

Experiments with isolated perfused beating hearts are frequently used in cardiac animal experiments. These experiments are necessary to characterize radionuclide tracer kinetic parameters such as first-pass extraction and tracer washout. The isolated heart setting enables this analysis without interference by systemic tracer recirculation (18). Additionally, variable environmental conditions can be tested including changes in flow rate, work load, glucose, and insulin concentrations. The degree of control is a unique feature of this ex vivo approach in comparison with in vivo experiments
(19). Furthermore, blocking experiments targeting specific processes at the molecular level can identify detailed and specific mechanisms of tracer handling in the heart. For example, DeGrado et al. characterized a ${ }^{11} \mathrm{C}$-labeled sympathetic nerve PET tracer, meta-hydroxyephedrine (HED), using this ex vivo technique. Desipramine, a neural uptake-1 blocker, reduced cardiac HED uptake, indicating high HED specificity for the cardiac nerve terminals. Furthermore, there was increased washout of HED after the administration of a desipramine chase, further confirming the importance of the uptake-1 mechanism for tracer retention (10). Although isolated perfused small-animal heart experiments are a well-established and unique technique for characterizing tracer kinetics, it should be noted that there are potential limitations. Small-animal hearts may have physiologic differences in comparison to human hearts, and small-animal experimental results may not fully extrapolate to clinical in vivo PET imaging.

Dedicated $\gamma$-detector systems are one of the widely used approaches to measure cardiac radiolabeled tracer activity in isolated perfused heart experiments. These are mostly custom-built inhouse $\gamma$-coincidence counting systems, which are equipped with 2 lead collimated bismuth germinate scintillation crystals $(6,7)$. This approach can measure only the entire tracer activity for the heart and provides no regional or segmental data. $\beta$-detector probes would potentially allow for characterizing regional tracer kinetics. However, such $\beta$ probes with plastic scintillators identify $\beta$ activity only in tissues near the probe because of limited travel distance of positrons within the tissue before annihilation (positron range

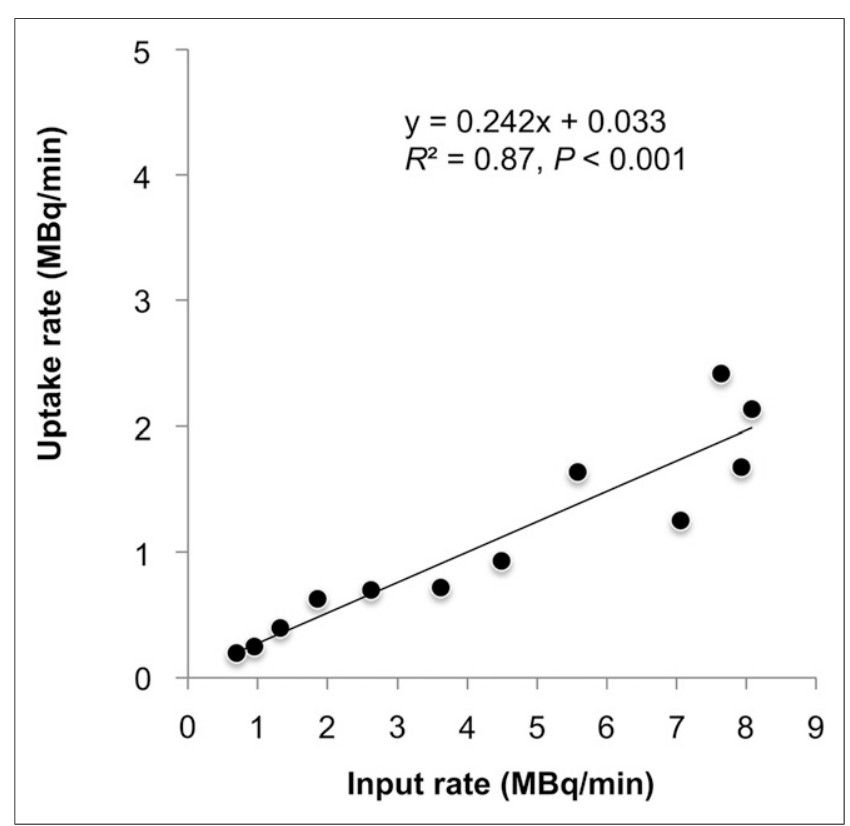

FIGURE 3. Linear regression plot of tracer input and uptake rate. Uptake rate indicates slope of time-activity curve calculated by linear fitting. 


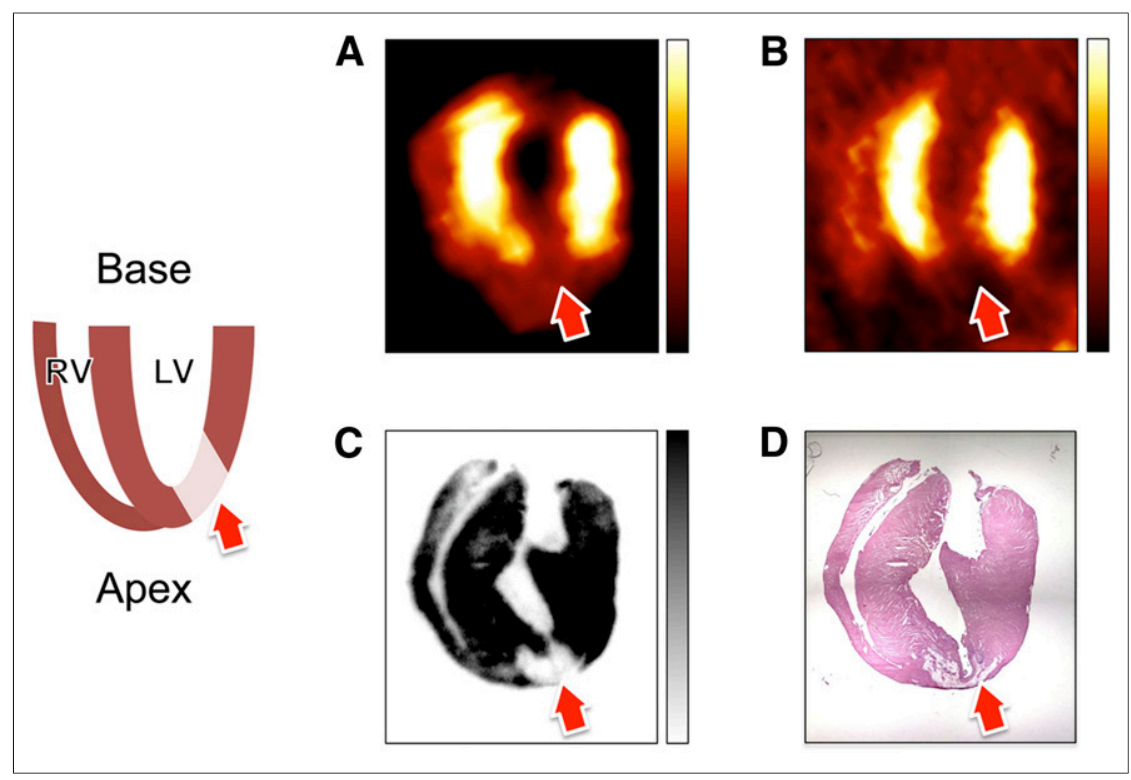

FIGURE 4. Horizontal long-axis images of myocardial infarction heart with ex vivo isolated perfused heart PET (A), in vivo PET (B), autoradiography of tissue slices (C), and histology of hematoxylin and eosin staining (D). Tracer uptake defects correspond with localization of scar tissue identified by histology (arrows). LV = left ventricle; RV = right ventricle.

of ${ }^{18} \mathrm{~F}$ in water is approximately $1.0 \mathrm{~mm}$ ). Lerch et al. introduced a $\beta$-probe method for detecting regional time-activity curves in open-chest dog hearts (20). Although this approach may be promising for regional tracer analysis in isolated perfused heart experiments, it has several potential limitations. Primarily, it is not clear if this $\beta$-probe method can be used for small-rodent hearts. Furthermore, the measurement is limited to a single region targeted by the detector, and whole cardiac counts cannot be analyzed simultaneously. More recently, tomographic approaches with isolated perfused heart experiments have been introduced by Garlick et al. $(21,22)$. They created a dedicated isolated perfused heart PET system with a single ring containing 72 lutetium oxyorthosilicate scintillation crystals coupled to optical fibers connected to 3 photomultiplier tubes. This system was designed to be compatible with simultaneous MR imaging. However, the field of view of this system is limited to a $25-\mathrm{mm}$ diameter and a single approximately 1-mm slice. Using this dedicated detector device, they demonstrated excellent tomographic ${ }^{18} \mathrm{~F}-\mathrm{FDG}$ images of the isolated perfused heart. However, this technique was limited by the lack of ability to measure total cardiac counts. In this present study, a commercially available small-animal PET system was coupled with a Langendorff isolated perfused heart apparatus to conduct experiments investigating tissue tracer kinetics. We demonstrated the feasibility of reconstructing tomographic images of the entire rodent isolated perfused heart and accurate quantification of whole cardiac activity measurements. This combined methodology adds value by allowing for tomographic regional tracer analysis and is more cost-effective than traditional in-house $\gamma$-detector systems. We believe this study demonstrates that our combination method is preferable and more effective than traditional ex vivo techniques.

Because we used reconstructed tomographic PET images for measuring counts of the isolated hearts, there are several potential sources of artifact for absolute quantification including scatter, motion, and attenuation. These limitations are somewhat miti- gated in the setting of the isolated perfused heart. We expect less influence by scatter artifacts in comparison with in vivo imaging given that adjacent structural radiotracer activity is nonexistent. To minimize error caused by region-of-interest selection and attenuation artifacts, we used a small (rat heart size) ${ }^{18} \mathrm{~F}$-filled phantom that contained scatter effects similar to the isolated heart. This phantom acts as a reference to calibrate count differences measured between PET and those measured by the $\gamma$ well counter. Further compensation approaches to improve the accuracy could be applied such as electrocardiogram-gated imaging to reduce motion artifacts and attenuation correction by performing additional transmission scans.

\section{CONCLUSION}

A unified ex vivo isolated perfused heart and small-animal PET system allows for simple and accurate global and regional quantification of myocardial tissue tracer distribution and kinetics. This novel assay could be useful for the characterization of myocardial PET tracers.

\section{DISCLOSURE}

The costs of publication of this article were defrayed in part by the payment of page charges. Therefore, and solely to indicate fact, this article is hereby marked "advertisement" in accordance with 18 USC section 1734. This study was supported by CHFC, University of Würzburg and Alexander von Humboldt Foundation. No other potential conflict of interest relevant to this article was reported.

\section{ACKNOWLEDGMENTS}

We are deeply grateful to Dr. Michael Lassmann who provided helpful comments and suggestions. Special thanks also go to Dr. Thorsten Winter who provided technical help.

\section{REFERENCES}

1. Bengel FM, Higuchi T, Javadi MS, Lautamaki R. Cardiac positron emission tomography. J Am Coll Cardiol. 2009;54:1-15.

2. Higuchi T, Bengel FM. Cardiovascular nuclear imaging: from perfusion to molecular function: non-invasive imaging. Heart. 2008;94:809-816.

3. Bell RM, Mocanu MM, Yellon DM. Retrograde heart perfusion: the Langendorff technique of isolated heart perfusion. J Mol Cell Cardiol. 2011;50:940-950.

4. Fukushima K, Momose M, Kondo C, Higuchi T, Kusakabe K, Hagiwara N. Myocardial ${ }^{99 \mathrm{~m}} \mathrm{Tc}$-sestamibi extraction and washout in hypertensive heart failure using an isolated rat heart. Nucl Med Biol. 2010;37:1005-1012.

5. Fukushima K, Momose M, Kondo C, Kusakabe K, Kasanuki H. Myocardial kinetics of ${ }^{201}$ thallium, ${ }^{99 \mathrm{~m}} \mathrm{Tc}$-tetrofosmin, and ${ }^{99 \mathrm{~m}} \mathrm{Tc}$-sestamibi in an acute ischemiareperfusion model using isolated rat heart. Ann Nucl Med. 2007;21:267-273.

6. Momose M, Reder S, Raffel DM, et al. Evaluation of cardiac beta-adrenoreceptors in the isolated perfused rat heart using (S)-1 ${ }^{11}$ C-CGP12388. J Nucl Med. 2004;45:471-477.

7. Huisman MC, Higuchi T, Reder S, et al. Initial characterization of an ${ }^{18} \mathrm{~F}$-labeled myocardial perfusion tracer. J Nucl Med. 2008;49:630-636.

8. Simões MV, Miyagawa M, Reder S, et al. Myocardial kinetics of reporter probe ${ }^{124}$ I-FIAU in isolated perfused rat hearts after in vivo adenoviral transfer of 
herpes simplex virus type 1 thymidine kinase reporter gene. J Nucl Med. 2005; 46:98-105.

9. Nguyen NT, DeGrado TR, Chakraborty P, Wieland DM, Schwaiger M. Myocardial kinetics of carbon-11-epinephrine in the isolated working rat heart. $\mathrm{J} \mathrm{Nucl}$ Med. 1997;38:780-785.

10. DeGrado TR, Hutchins GD, Toorongian SA, Wieland DM, Schwaiger M. Myocardial kinetics of carbon-11-meta-hydroxyephedrine: retention mechanisms and effects of norepinephrine. J Nucl Med. 1993;34:1287-1293.

11. Visser EP, Disselhorst JA, Brom M, et al. Spatial resolution and sensitivity of the Inveon small-animal PET scanner. J Nucl Med. 2009;50:139-147.

12. Shoghi KI. Quantitative small animal PET. $Q$ J Nucl Med Mol Imaging. 2009;53:365-373.

13. Herrmann K, Dahlbom M, Nathanson D, et al. Evaluation of the Genisys4, a bench-top preclinical PET scanner. J Nucl Med. 2013;54:1162-1167.

14. Higuchi T, Nekolla SG, Jankaukas A, et al. Characterization of normal and infarcted rat myocardium using a combination of small-animal PET and clinical MRI. J Nucl Med. 2007;48:288-294.

15. Guide for the Care and Use of Laboratory Animals. NIH publication no. 85-23. Bethesda, MD: U.S. National Institutes of Health; 1985, revised 1996.
16. Loening AM, Gambhir SS. AMIDE: a free software tool for multimodality medical image analysis. Mol Imaging. 2003;2:131-137.

17. Higuchi T, Rischpler C, Fukushima K, et al. Targeting of endothelin receptors in the healthy and infarcted rat heart using the PET tracer ${ }^{18} \mathrm{~F}-\mathrm{FBzBMS} . J \mathrm{Nucl}$ Med. 2013;54:277-282.

18. Depre C. Isolated working heart: description of models relevant to radioisotopic and pharmacological assessments. Nucl Med Biol. 1998;25:711713.

19. Huang SC, Williams BA, Krivokapich J, Araujo L, Phelps ME, Schelbert HR. Rabbit myocardial ${ }^{82} \mathrm{Rb}$ kinetics and a compartmental model for blood flow estimation. Am J Physiol. 1989;256:H1156-H1164.

20. Lerch RA, Ambos HD, Bergmann SR, Sobel BE, Ter-Pogossian MM. Kinetics of positron emitters in vivo characterized with a beta probe. Am J Physiol. 1982;242:H62-H67.

21. Garlick PB, Marsden PK, Cave AC, et al. PET and NMR dual acquisition (PANDA): applications to isolated, perfused rat hearts. NMR Biomed. 1997;10: $138-142$.

22. Garlick PB, Medina RA, Southworth R, Marsden PK. Differential uptake of FDG and DG during post-ischaemic reperfusion in the isolated, perfused rat heart. Eur J Nucl Med. 1999;26:1353-1358. 